\title{
Hyperfunction Quantum Field Theory
}

\author{
S. Nagamachi \\ Department of Mathematics, Faculty of Engineering, Tokushima University, Tokushima 770, Japan \\ N. Mugibayashi \\ Department of Electrical Engineering, Kobe University, Rokkodai, Kobe 657, Japan
}

\begin{abstract}
The quantum field theory in terms of Fourier hyperfunctions is constructed. The test function space for hyperfunctions does not contain $C^{\infty}$ functions with compact support. In spite of this defect the support concept of $H$-valued Fourier hyperfunctions allows to formulate the locality axiom for hyperfunction quantum field theory.
\end{abstract}

\section{§1. Introduction}

In the usual framework of axiomatic quantum field theory, founded by Wightman [1], one assumes fields to be operator-valued tempered distributions. For nonrenormalizable interactions, however, the fields seem no longer remain tempered [2]. Several attempts have been made to extend Wightman's axioms for the quantum field theory so as to include a wider class of fields [2-4]. On the other hand, the recent development of the Euclidean field theory reveals that the temperedness of fields shows some inconvenience on coming back to the relativistic quantum field theory [5].

From the mathematical point of view the extension of Wightman's axioms starts with replacing the test function space $\mathscr{S}$ of tempered fields, the Schwartz space of rapidly decreasing functions, by its suitable dense subspace. In carrying through this program the most obstructive will be the axiom concerning the localizability of fields. The test function spaces considered so far by several authors contain $C^{\infty}$ functions with compact support in configuration and/or momentum spaces. Hence the localizability of the fields has been preserved quite naturally in some way or other.

In the present paper we wish to formulate the quantum field theory in terms of Fourier hyperfunctions. The space of Fourier hyperfunctions is the dual of the space of rapidly decreasing holomorphic functions [6]. One of the characteristics of the latter space is that it is topologically invariant under Fourier transformations as is the case for the space $\mathscr{S}$. But since our space contains no functions of compact support, we are not allowed to state the locality of the field in the usual sense. In order to remedy this difficulty one of the present authors (S.N.), 
in collaboration with Ito, has developed the theory of vector-valued Fourier hyperfunctions [7]. By the help of a remarkable notion of the "support of hyperfunctions" we succeed to formulate the locality of hyperfunction fields. We note that our test function space is the smallest of all that have been proposed up to the present.

After some mathematical preliminaries we discuss in what follows the axioms for hyperfunction quantum field theory, main properties of Wightman Fourier hyperfunctions, and the reconstruction theorem, wherein our principal attention will be turned to the "locality" problem. In a forthcoming paper we shall study the Euclidean field theory for hyperfunction fields.

\section{§ 2. Test Function Spaces}

We adopt the standard notations for $n$-tuple of numbers. Thus, let $x \in \mathbb{R}^{n}$, $k=\left(k_{1}, \ldots, k_{n}\right)$ and $l=\left(l_{1}, \ldots, l_{n}\right)$ be $n$-tuples of nonnegative integers, then $x^{k}=x_{1}^{k_{1}} \ldots x_{n}^{k_{n}}$ and $D^{l}=\partial^{|l|} / \partial x_{1}^{l_{1}} \ldots \partial x_{n}^{l_{n}}$. Here $|l|=l_{1}+\ldots+l_{n}$; more generally $|z|=\left|z_{1}\right|+\ldots+\left|z_{n}\right|$ if $z \in \mathbb{C}^{n}=\mathbb{R}^{n} \times i \mathbb{R}^{n}$. The inequality $k>l$ means that $k_{j}>l_{j}$ for $1 \leqq j \leqq n$.

2.1. $\mathscr{M}_{\omega}$

The test function space for Jaffe's class of ultra-distributions [2] is characterized by a real-valued function $\omega(s)$ on $[0, \infty)$, called a Jaffe indicatrix, which satisfies the following conditions [8]:

a) (Regularity) $\exp (\omega(s))$ is a real entire function:

$$
e^{\omega(s)}=\sum_{r=0}^{\infty} a_{r} s^{r}
$$

and $a_{0}=1, a_{r} \geqq 0(r=1,2,3, \ldots)$.

b) (Subadditivity) $\omega(s+t) \leqq \omega(s)+\omega(t)$ for all $s, t \in[0, \infty)$.

c) (Carleman's criterion)

$\int_{0}^{\infty} \frac{\omega\left(s^{2}\right)}{1+s^{2}} d s<\infty$

d) (Nuclearity) $2 \omega(s) \leqq \omega(A s)+C$ for some constants $A, C$.

To avoid trivial cases we assume the Jaffe indicatrix to satisfy one more condition [8]:

e) $\omega(s) \geqq \log (1+s)$.

Consider a set of seminorms for functions $f(x)$ on $\mathbb{R}^{n}$

$$
\|f\|_{l, \lambda}^{(\omega)}=\sup _{x} e^{\lambda \omega\left(\|x\|^{2}\right)}\left|D^{l} f(x)\right|,
$$

where $\lambda>0, l$ is an $n$-tuple of nonnegative integers and $\|x\|$ is the Euclidean norm in $\mathbb{R}^{n}$. The space $\mathscr{M}_{\omega}$ is defined by

$$
\mathscr{M}_{\omega}=\left\{f ;\|f\|_{\lambda, l}^{(\omega)}<\infty \text { for every } l, \lambda\right\} .
$$

It is evident that $\mathscr{M}_{\omega} \supset \mathscr{D}$, the space of $C^{\infty}$ functions with compact support. The condition e) above assures that $\mathscr{M}_{\omega} \subset \mathscr{S}$, the Schwartz space of rapidly decreasing 
functions. Carleman's criterion c) implies that the Fourier transform of $\mathscr{M}_{\omega}$, $\mathscr{C}_{\omega}=\mathscr{F}\left(\mathscr{M}_{\omega}\right)$, contains functions of compact support.

\section{2. $\mathscr{S}_{1}$}

The space $\mathscr{S}_{1, A}$ consists of those functions $f(x)$ on $\mathbb{R}^{n}$ which satisfy the inequalities

$$
\left|x^{k} D^{l} f(x)\right| \leqq C_{l \delta}(A+\delta)^{|k|} k^{k}
$$

for any $\delta>0$. It is known [9] that the space $\mathscr{S}_{1, A}$ is a countably normed spaee with an infinite set of norms

$$
\|f\|_{m, p}=\sup _{x,|l| \leqq p}\left|D^{l} f(x)\right| e^{(1 / m)(1-1 / p)|x|},
$$

where $p=2,3, \ldots$ and $m=e A$. The topology of $\mathscr{S}_{1, A}$ is given by the norms $\left\{\|\cdot\|_{e A, p}\right\}_{p=2}^{\infty}$. The space $\mathscr{S}_{1}$ is a union of $\mathscr{S}_{1, A}$ and the inductive limit topology is introduced in it.

We shall show that $\mathscr{S}_{1} \subset \mathscr{M}_{\omega}$ for any Jaffe indicatrix $\omega$. Let us begin by verifying the limit

$$
\limsup _{s \rightarrow \infty} \frac{\omega\left(s^{2}\right)}{s}=0 \text {. }
$$

In fact, if one assumes the contrary, there would be a sequence of increasing positive numbers tending to infinity, $\left\{\alpha_{n}\right\}$, and $\varepsilon>0$ such that $\omega\left(\alpha_{n}\right)>\varepsilon \alpha_{n}$. By the condition a) the indicatrix $\omega\left(s^{2}\right)$ is monotone increasing and hence we have $\omega\left(s^{2}\right)>\varepsilon \alpha_{n}$ for every $s \geqq \alpha_{n}$. Then it is easy to see that for any $\alpha_{n}>1$

$$
\int_{\alpha_{n}}^{\infty} \frac{\omega\left(s^{2}\right)}{1+s^{2}} d s \geqq \frac{\varepsilon}{2},
$$

which contradicts Carleman's criterion c). Therefore we have $\omega\left(s^{2}\right)<a|s|$ for any $a>0$ and a sufficiently large $|s|$. Combining with the conditions a) and b) this means that

$$
e^{\omega\left(\|x\|^{2}\right)} \leqq C_{a} e^{a|x|},
$$

which implies $\mathscr{S}_{1} \subset \mathscr{M}_{\omega}$.

\section{3. $\mathscr{S}_{1}^{1}$}

The space $\mathscr{S}_{1, A}^{1, B}$ consists of those functions $f(x)$ on $\mathbb{R}^{n}$ which satisfy the inequalities

$$
\left|x^{k} D^{l} f(x)\right| \leqq C_{\delta \varrho}(A+\delta)^{|k|}(B+\varrho)^{|l|} k^{k} l^{l}
$$

for $\delta, \varrho=1 / 2,1 / 3, \ldots$ [9]. The space $\mathscr{S}_{1, A}^{1, B}$ is a countably normed space with an infinite set of norms

$$
\left.\|f\|_{A, B ; \delta, \varrho}=\sup _{x, k, l} \mid x^{k} D^{l} f(x)\right) \mid /(A+\delta)^{|k|}(B+\varrho)^{|l|} k^{k} l^{l} .
$$

The space $\mathscr{S}_{1}^{1}$ is defined by $\mathscr{S}_{1}^{1}=\operatorname{ind} \lim _{A, B} \mathscr{S}_{1, A}^{1, B}$. This space can also be regarded as an inductive limit of Banach spaces. Let us define the space $\mathscr{T}_{A}^{B}$ by

$$
\mathscr{T}_{A}^{B}=\left\{f ;\|f\|_{A, B}<\infty\right\},
$$


where

$$
\|f\|_{A, B}=\sup _{x, k, l}\left|x^{k} D^{l} f(x)\right| / A^{|k|} B^{|l|} k^{k} l^{l} .
$$

From the obvious relations

$$
\|f\|_{A, B} \geqq\|f\|_{A, B ; \delta, \varrho} \geqq\|f\|_{A+1, B+1},
$$

we at once conclude that ind $\lim _{A, B} \mathscr{T}_{A}^{B}=\mathscr{S}_{1}^{1}[10]$.

The spaces $\mathscr{S}_{1}$ and $\mathscr{S}_{1}^{1}$ are both of type $S$. The spaces of type $S$ are studied in detail in a textbook by Gel'fand and Shilov [9]. Among other properties of the space $\mathscr{S}_{1}^{1}$, the followings are worthy to be noticed.

$1^{\circ} \mathscr{S}_{1}^{1} \subset \mathscr{S}_{1}$.

$2^{\circ} \mathscr{S}_{1}^{1}$ is a nuclear space [10].

$3^{\circ} \mathscr{F}\left(\mathscr{S}_{1}^{1}\right)=\mathscr{S}_{1}^{1}$.

\section{4. $\mathscr{P}_{*}$}

$\mathcal{O}_{c}^{m}$ is the Banach space of those functions which are holomorphic in $|\operatorname{Im} z|<1 / m$ and continuous in $|\operatorname{Im} z| \leqq 1 / m$. The norm of this space is defined by

$$
\|f\|_{m}=\sup _{|\operatorname{Im} z| \leqq 1 / m}|f(z)| e^{|z| / m} .
$$

The space of rapidly decreasing holomorphic functions $\mathscr{P}_{*}$ is the inductive limit of the Banach spaces $\mathcal{O}_{c}^{m}: \mathscr{P}_{*}=\operatorname{ind} \lim _{m} \mathcal{O}_{c}^{m}$.

Proposition 2.1. The spaces $\mathscr{P}_{*}$ and $\mathscr{S}_{1}^{1}$ are isomorphic.

Proof. It suffices to show that for each $\mathscr{T}_{A}^{B}$ (resp. $\mathcal{O}_{c}^{m}$ ) there exists an $\mathcal{O}_{c}^{m}$ (resp. $\mathscr{T}_{A}^{B}$ ) such that $\mathscr{T}_{A}^{B} \subset \mathcal{O}_{c}^{m}$ (resp. $\mathcal{O}_{c}^{m} \subset \mathscr{T}_{A}^{B}$ ) and thereby the embedding is continuous.

In order to avoid the notational complication we prove the proposition only in the case $n=1$. The generalization of the proof to an arbitrary $n$ is straightforward. Gel'fand and Shilov [9] state that $\|f\|_{A, B}=M<\infty$ implies $\left|D^{l} f(x)\right| \leqq$ $C_{1} M \exp (-|x| / e A) B^{l} l^{l}$ with $C_{1}=\exp (e / 2)$. This in turn assures that $f(x)$ can be analytically continued to $|\operatorname{Im} z|<1 / e B$ and therein the estimate

$$
|f(z)| \leqq C_{2} M \exp (-|x| / e A)(1-e B|\operatorname{Im} z|)^{-1}
$$

holds for some constant $C_{2}$ independent of $A$ and $B$. If we take $m>\max (e A, e B)$, then $f(z)$ is analytic in $|\operatorname{Im} z|<1 / m$ and satisfies $|f(z)| \leqq C_{3} M \exp (-|z| / m)$, where $C_{3}$ is a constant depending on $B$. Thus we have $\|f\|_{m} \leqq C_{3}\|f\|_{A, B}$, which means that $\mathscr{T}_{A}^{B} \subset \mathcal{O}_{c}^{m}$ with $m>\max (e A, e B)$ and the embedding is continuous.

The remaining part of the proof is carried out on the basis of Theorem 4 on p. 223 of Gel'fand and Shilov's textbook [9]. Let $\|f\|_{m}=M<\infty$, then $f(z)$ is analytic in $|\operatorname{Im} z| \leqq a<1 / m$ and satisfies $|f(z)| \leqq M \exp (-a|z|)$. The abovementioned theorem states that there exists a constant $B>1 / a$ and the estimate $\left|D^{l} f(x)\right| \leqq C_{1} M B^{l} l^{l} \exp (-a|x|)$ holds, where $C_{1}$ is a constant depending on $a$. By means of the inequality $\exp (-|\xi| / e) \leqq \inf _{k} k^{k} /|\xi|^{k}$, which also is verified in [9], and by putting $A=1 /$ ae we finally obtain $\|f\|_{A, B} \leqq C_{1}\|f\|_{m}$. This means that $\mathcal{O}_{c}^{m} \subset \mathscr{T}_{A}^{B}$ with $A>m / e, B>m$ and the embedding is continuous. The proof of Proposition 2.1 is thus completed. 
In closing this section we summarize the test function spaces studied above in the following scheme:

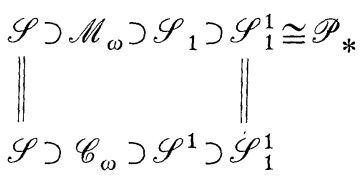

The spaces on the lower line are the Fourier transform of corresponding spaces on the upper line ${ }^{1}$. The space $\mathscr{S}^{1}$ is such that each $f(x) \in \mathscr{S}^{1}$ has an analytic continuation in a certain complex neighbourhood of $\mathbb{R}^{n}$. The spaces that contain functions of compact support are $\mathscr{S}, \mathscr{M}_{\omega}, \mathscr{C}_{\omega}$, and $\mathscr{S}_{1}$.

\section{§ 3. $\boldsymbol{H}$-valued Fourier Hyperfunctions}

In this section we give the definition of $H$-valued Fourier hyperfunctions and make mention of their main properties, especially the concept of the "support of Fourier hyperfunctions", which becomes important in formulating the locality axiom of the quantum field theory. We shall either omit or sketch only briefly the proof of most of the statements. For more details we refer to papers of Kawai [6], and Ito and Nagamachi [7].

Recently the theory of vector-valued hyperfunctions has been presented by Ion and Kawai [11]. In constructing the theory they used the method of "soft analysis" in parallel with Sato's theory of hyperfunctions. A similar theory has also been developed by Ito and Nagamachi [7] by the method analogous to Kawai's construction of the Fourier hyperfunctions [6].

Let $\mathbb{D}^{n}$ denote the compactification of $\mathbb{R}^{n}: \mathbb{D}^{n}=\mathbb{R}^{n} \sqcup \mathbb{S}_{\infty}^{n-1}$, where $\mathbb{S}_{\infty}^{n-1}$ is an $(n-1)$-dimensional sphere at infinity. To each $x \in \mathbb{R}^{n}-\{0\}$ we associate a point $x_{\infty}$ on $\mathbb{S}_{\infty}^{n-1}$ such that the point $x$ lies on the ray connecting $x_{\infty}$ and the origin. We identify $\mathbb{S}^{n-1}$, an $(n-1)$-dimensional sphere centered at the origin, with $\mathbb{R}^{n}-\{0\} / \mathbb{R}^{+}$, where $\mathbb{R}^{+}=\{x \in \mathbb{R} ; x>0\}$. A natural topology is given to the space $\mathbb{D}^{n}$ : (i) If a point $x$ belongs to $\mathbb{R}^{n}$ a fundamental system of neighbourhoods of $x$ is the set of all open balls containing the point $x$. (ii) If a point $x$ belongs to $\mathbb{S}_{\infty}^{n-1}$ we write $x=y_{\infty}$ and let $y$ be the corresponding point on $\mathbb{S}^{n-1}$. A fundamental system of neighbourhoods of $x$ is given by $\left\{(C+a) \cup C_{\infty} ; C_{\infty} \ni y_{\infty}\right\}$, where $C$ is an open cone generated by some open neighbourhood of $y$ in $\mathbb{S}^{n-1}$ with its vertex at the origin, $a$ is some vector in $\mathbb{R}^{n}$, so that $C+a$ is a cone with its vertex at $a$, and $C_{\infty}$ signifies the points at infinity of that cone. In what follows we use the notation $\mathbb{Q}^{n}=\mathbb{D}^{n} \times i \mathbb{R}^{n}$.

Let us begin with the description of (scalar-valued) Fourier hyperfunctions.

\footnotetext{
1 We are grateful to the referee who pointed out that Jaffe modified his definitions of the test functions to give a formulation which is symmetric under Fourier transformations, while preserving all the other properties of strictly localizable fields. This was done by replacing $\mathscr{M}_{\omega},(2.2)$, by a space equipped with the norm given by
}

$$
\sum_{r=0}^{\infty} a_{r} \sum_{|k|+|l|=r}\left|x^{k} D^{l} f(x)\right|_{\infty},
$$

where $a_{r}$ 's are the coefficients in the regularity condition a) of the Jaffe indicatrix. 
Definition 3.1. (The sheaf of slowly increasing holomorphic functions.) We denote by $\tilde{\mathcal{O}}$ the sheaf whose section module $\tilde{\mathcal{O}}(\Omega)$ over an open set $\Omega$ in $\mathbb{Q}^{n}$ is the set of all holomorphic functions $f(z)\left(\in \mathcal{O}\left(\Omega \cap \mathbb{C}^{n}\right)\right)$ such that $\sup _{z \in K \cap \mathbb{C}^{n}}|f(z)| e^{-\varepsilon|z|}<\infty$ for any positive $\varepsilon$ and any compact set $K$ in $\Omega$. It is clear that the presheaf $\{\tilde{\mathcal{O}}(\Omega)\}$ constitutes a sheaf over $\mathbb{Q}^{n}$.

Definition 3.2. (The sheaf of rapidly decreasing holomorphic functions.) We denote by $\underset{\mathcal{O}}{\mathcal{Q}}$ the sheaf whose section module $\underset{\sim}{\mathcal{Q}}(\Omega)$ over an open set $\Omega$ in $\mathbb{Q}^{n}$ is the set of all holomorphic functions $f(z)\left(\in \mathcal{O}\left(\Omega \cap \mathbb{C}^{n}\right)\right)$ such that for any compact set $K$ in $\Omega$ there exists some positive constant $\delta_{K}$ and the estimate $\sup _{z \in K \cap \mathbb{C}^{n}}|f(z)| e^{\delta_{K}|z|}<\infty$ holds.

Definition 3.3. [Topology of $\mathcal{O}(K)$.] Let $K$ be a compact set in $\mathbb{D}^{n}$. We give $\mathcal{Q}(K)$ the inductive limit topology ind $\lim \mathcal{O}_{c}^{m}\left(U_{m}\right)$, where $\left\{U_{m}\right\}$ is a fundamental system of neighbourhoods of $K$ in $\mathbb{Q}^{n}$, satisfying $U_{m} \gg U_{m+1}$, and $\mathcal{O}_{c}^{m}\left(U_{m}\right)$ is the Banach space of all holomorphic functions $f(z)\left(\in \mathcal{O}\left(U_{m} \cap \mathbb{C}^{n}\right)\right)$ that are continuous in $\bar{U}_{m} \cap \mathbb{C}^{n}$ and for which $|f(z)| \leqq C e^{-|z| / m}$ holds for some constant $C$ (depending on $f)$. The norm of $\mathcal{O}_{c}^{m}\left(U_{m}\right)$ is defined by $\|f\|_{m}=\sup _{z \in U_{m} \cap \mathbb{C}^{n}}|f(z)| e^{|z| / m}$.

With this topology $\mathscr{Q}(K)$ becomes a DFS-space (a dual Fréchet-Schwartz space). When $\mathbb{D}^{n}$ itself is taken as $K, \mathcal{Q}\left(\mathbb{D}^{n}\right)$ is evidently identical with $\mathscr{P}_{*}$ introduced in $\S 2.4$. Then we have

Proposition 3.4. $\mathscr{Q}\left(\mathbb{D}^{n}\right)$ is a nuclear space.

Proposition 3.5. $\underset{n}{\bigotimes} \mathcal{Q}(\mathbb{D})$ is dense in $\underset{\sim}{\mathcal{Q}}\left(\mathbb{D}^{n}\right)$ and $\underset{n}{\hat{\bigotimes}} \mathcal{O}(\mathbb{D})=\mathscr{Q}\left(\mathbb{D}^{n}\right)$.

Proof. Mityagin [10] proved similar propositions for $\mathscr{S}_{1}^{1}$. Because of the isomorphism between $\underset{\sim}{\mathscr{O}}\left(\mathbb{D}^{n}\right)$, or $\mathscr{P}_{*}$, and $\mathscr{S}_{1}^{1}$, Proposition 2.1 , his argument equally apply to $\mathcal{Q}\left(\mathbb{D}^{n}\right)$.

Proposition 3.6. A separately continuous multilinear form $M$ on $[\underset{Q}{Q}(\mathbb{D})]^{n}$ uniquely defines an element of $\left(\mathcal{Q}\left(\mathbb{D}^{n}\right)\right)^{\prime}$, the dual space of $\mathcal{Q}\left(\mathbb{D}^{n}\right)$, such that

$$
M\left(\phi_{1}, \ldots, \phi_{n}\right)=F\left(\phi_{1} \otimes \ldots \otimes \phi_{n}\right) \quad \text { for } \quad \phi_{j} \in \mathcal{O}_{(}(\mathbb{D}), \quad j=1,2, \ldots, n .
$$

Proof. Since $\mathcal{Q}\left(\mathbb{D}^{k}\right)$ is a DFS-space, $\mathcal{Q}\left(\mathbb{D}^{k}\right)$ is a strong dual of a reflexive Fréchet space $[6,12]$. By a multilinear version of Theorem 41.1 of Treves [13] we can state that the separately continuous multilinear form on $[\mathcal{Q}(\mathbb{I D})]^{n}$ is continuous. Since $\mathcal{Q}\left(\mathbb{D}^{k}\right)$ is nuclear and $\otimes \mathcal{Q}(\mathbb{D})=\mathscr{\mathcal { O }}\left(\mathbb{D}^{n}\right)$ by the preceding propositions, the form $M$

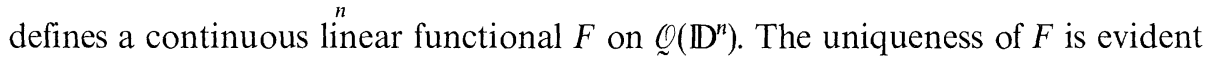
from the fact that $\bigotimes_{n} \mathcal{Q}(\mathbb{D D})$ is dense in $\underset{\sim}{\mathcal{O}}\left(\mathbb{D}^{n}\right)$.

Definition 3.7. Let $\Omega$ be an open set in $\mathbb{D}^{n}$. We choose an open set $V$ in $\mathbb{Q}^{n}$ which contains $\Omega$ as a relatively closed set and define $\mathscr{R}(\Omega)$, the space of Fourier hyperfunctions over $\Omega$, by the cohomology $H_{\Omega}^{n}(V, \tilde{\mathcal{O}})$. (By the excision theorem the space $\mathscr{R}(\Omega)$ is independent of the choice of $V$.)

Proposition 3.8. When $K$ is a compact set in $\mathbb{D}^{n}$, we have $H_{K}^{n}(V, \tilde{\mathcal{O}}) \cong(\mathscr{Q}(K))^{\prime}$, especially $\mathscr{R}\left(\mathbb{D}^{n}\right) \cong\left(\mathscr{Q}\left(\mathbb{D}^{n}\right)\right)^{\prime}$.

Proof. See Kawai [6]. 
Now we are in a position to generalize the above definitions and propositions for Fourier hyperfunctions to the case when Fourier hyperfunctions take on their values in a separable Hilbert space $H$.

Definition 3.1'. We denote by ${ }^{H} \tilde{\mathcal{O}}$ the sheaf whose section module ${ }^{H} \tilde{\mathcal{O}}(\Omega)$ over an open set $\Omega$ in $\mathbb{Q}^{n}$ is the set of all $H$-valued holomorphic functions $f(z)$ such that for any positive $\varepsilon$ and any compact set $K$ in $\Omega$, the estimate

$$
\sup _{z \in K \cap \mathbb{C}^{n}}\|f(z)\| e^{-\varepsilon|z|}<\infty
$$

holds, where $\|\cdot\|$ stands for the norm in $H$.

Definition $3.2^{\prime}$. We denote by ${ }^{H} \mathcal{Q}$ the sheaf whose section module ${ }^{H} \mathcal{Q}(\Omega)$ over an open set $\Omega$ in $\mathbb{Q}^{n}$ is the set of all $H$-valued holomorphic functions $f(z)$ such that for any compact set $K$ in $\Omega$ there exists some positive constant $\delta_{K}$ and the estimate $\sup _{z \in K \cap \mathbb{C}^{n}}\|f(z)\| e^{\delta_{K}|z|}<\infty$ holds.

Definition 3.7'. Let $\Omega$ be an open set in $\mathbb{D}^{n}$. We choose an open set $V$ in $\mathbb{Q}^{n}$ which contains $\Omega$ as a relatively closed set and define ${ }^{H} \mathscr{R}(\Omega)$, the space of $H$-valued Fourier hyperfunctions over $\Omega$, by the cohomology $H_{\Omega}^{n}\left(V,{ }^{H} \tilde{\mathcal{O}}\right)$.

Theorem 3.8'. When $K$ is a compact set of $\mathbb{D}^{n}$ we have $H_{K}^{n}\left(V,{ }^{H} \tilde{\mathcal{O}}\right) \cong L(\mathcal{Q}(K), H)$, especially ${ }^{H} \mathscr{R}\left(\mathbb{D}^{n}\right) \cong L\left(\mathscr{Q}\left(\mathbb{D}^{n}\right), H\right)$, where $L(\mathcal{Q}(K), H)$ is the space of all continuous linear operators from $\mathcal{Q}(K)$ to $H$ equipped with the topology of bounded convergence.

The following corollary is evident from this theorem and Proposition 3.8.

Corollary 3.9. If $F$ is an $H$-valued Fourier hyperfunction, then $(\psi, F)$ for every $\psi \in H$ is a scalar-valued Fourier hyperfunction, where $(\cdot, \cdot)$ is the inner product in the Hilbert space $H$.

We now outline the argument leading to Theorem $3.8^{\prime}$. First let us show that an element $\Phi$ of $L\left(Q\left(\mathbb{D}^{n}\right), H\right)$ defines a slowly increasing $H$-valued holomorphic function $\phi$ on $(\mathbb{C}-\mathbb{R})^{n}$ and $\Phi$ can be considered as its boundary value.

Proposition 3.10. Let

$$
h_{z}(t)=(2 \pi i)^{-n} \prod_{j=1}^{n} \exp \left\{-\left(t_{j}-z_{j}\right)^{2}\right\} /\left(t_{j}-z_{j}\right)
$$

and $\phi(z)=\Phi\left(h_{z}\right)$ for $\Phi \in L\left(\underset{\sim}{\mathcal{O}}\left(\mathbb{D}^{n}\right), H\right)$, then $\phi(z)$ is a slowly increasing H-valued holomorphic function.

Proof. If $|\operatorname{Im} z|>\delta^{\prime}>\delta>0$ we have

$$
\begin{aligned}
& \sup _{|\operatorname{Im} t|<\delta}\left|\prod_{j} \exp \left\{-\left(t_{j}-z_{j}\right)^{2}\right\} /\left(t_{j}-z_{j}\right)\right| e^{\varepsilon|t|} \\
& \quad=\sup _{|\operatorname{Im}(z+u)|<\delta}\left|\prod_{j} \exp \left\{-\left(u_{j}\right)^{2}\right\} / u_{j}\right| e^{\varepsilon|z+u|} \leqq C_{\varepsilon} e^{\varepsilon|z|}
\end{aligned}
$$

for all positive $\varepsilon$. Thus it is found that $h_{z}(t)$ belongs to $\mathcal{Q}\left(\mathbb{D}^{n}\right)$ and $\phi(z)=\Phi\left(h_{z}\right)$ is well defined as a slowly increasing $H$-valued holomorphic function on $(\mathbb{C}-\mathbb{R})^{n}$. 
Proposition 3.11. Let $\phi(z)$ be as in the preceding proposition. Then for any $g \in \underset{\mathcal{Q}}{(}\left(\mathbb{D}^{n}\right)$ we have

$$
\int_{\Gamma_{1} \times \ldots \times \Gamma_{n}} \phi(z) g(z) d z=\Phi(g),
$$

where $d z=d z_{1} \ldots d z_{n}$ and $\Gamma_{j}$ is a path in the $j$-th complex plane consisting of two straight lines parallel to the real axis, one of which runs to the left below the real axis, the other to the right above the real axis.

Proof.

$$
\begin{aligned}
\Phi(g) & =\Phi\left(\int_{\Gamma_{1} \times \ldots \times \Gamma_{n}} h_{z} g(z) d z\right) \\
& =\int_{\Gamma_{1} \times \ldots \times \Gamma_{n}} \Phi\left(h_{z}\right) g(z) d z \\
& =\int_{\Gamma_{1} \times \ldots \times \Gamma_{n}} \Phi(z) g(z) d z .
\end{aligned}
$$

Let $V_{0}=\mathbb{Q}^{n}$ and $V_{j}=\left\{z \in \mathbb{Q}^{n} ; \operatorname{Im} z_{j} \neq 0\right\}$. Furthermore we put $V=\bigcap_{j=1}^{n} V_{j}$ and $\hat{V}_{j}=\bigcap_{i \neq j} V_{i}$. Propositions 3.10 and 3.11 provide a mapping $\kappa$ from $L\left(\mathcal{Q}\left(\mathbb{D}^{n}\right), H\right)$ to ${ }^{H} \tilde{O}(V)$ and a mapping $l$ from ${ }^{H} \tilde{\mathcal{O}}(V)$ to $L\left(\mathcal{Q}\left(\mathbb{D}^{n}\right), H\right)$, respectively. However, since the integral (3.2) vanishes for any $\phi \in \sum_{j=1}^{n} H \tilde{\mathcal{O}}\left(\hat{V}_{j}\right)$, namely for any $\phi=\sum_{j=1}^{n} \phi_{j}$ such that $\phi_{j} \in{ }^{H} \tilde{\mathcal{O}}\left(\hat{V}_{j}\right)$, the mapping $l$ is considered as a mapping from ${ }^{H} \tilde{\mathcal{O}}(V) / \sum_{j}{ }^{H} \tilde{\mathcal{O}}\left(\hat{V}_{j}\right)$ to $L\left(Q\left(\mathbb{D}^{n}\right), H\right)$. Correspondingly $\kappa$ is naturally considered as a mapping from $L\left(\mathcal{O}\left(\mathbb{D}^{n}\right), H\right)$ to ${ }^{H} \tilde{\mathcal{O}}(V) / \sum_{j}{ }^{H} \tilde{\mathcal{O}}\left(\hat{V}_{j}\right)$. The following proposition implies the isomorphism between $L\left(\mathcal{Q}\left(\mathbb{D}^{n}\right), H\right)$ and ${ }^{H} \tilde{\mathcal{O}}(V) / \sum_{j}{ }^{H} \tilde{\mathcal{O}}\left(\hat{V}_{j}\right)$.

Proposition 3.12. The mapping $l$ is injective, so $l$ and $\kappa$ are bijective.

Proof. For simplicity we assume $n=1$. Using the function $h_{z}(t),(3.1)$, for $n=1$ we consider integrals along the paths $\Gamma$ and $\Gamma^{\prime}$ in the complex $t$ plane. These paths are similar to $\Gamma_{j}$ in (3.2), but subject to the condition that $\Gamma$ goes round the point $t=z$ to the same side as the real axis, whereas $\Gamma^{\prime}$ to the side opposite to the real axis. By Cauchy's integral formula we have

$$
\phi(z)=\int_{\Gamma} \phi(t) h_{z}(t) d t-\int_{\Gamma^{\prime}} \phi(t) h_{z}(t) d t .
$$

Suppose $l(\phi)=0$, then $\int_{\Gamma} \phi(t) h_{z}(t) d t=0$ and $\phi(z)=-\int_{\Gamma^{\prime}} \phi(t) h_{z}(t) d t$ is an element of ${ }^{H} \tilde{\mathcal{O}}(Q)={ }^{H} \tilde{\mathcal{O}}\left(\hat{V}_{1}\right)$. That is $\phi=0$ as an element of ${ }^{H} \tilde{\mathcal{O}}(V) /{ }^{H} \tilde{\mathcal{O}}\left(\hat{V}_{1}\right)$. Hence $l$ is injective. Since $l \circ \kappa=$ identity, $l$ and $\kappa$ are bijective.

Remark. Let $W=\left\{V_{j}\right\}_{j=0}^{n}, W^{\prime}=\left\{V_{j}\right\}_{j=1}^{n}$. It can be shown [7] that $H_{\mathbb{D}^{n}}^{n}\left(\mathbb{Q}^{n},{ }^{H} \tilde{\mathcal{O}}\right) \cong$ $H^{n}\left(W, W^{\prime} ;{ }^{H} \tilde{\mathcal{O}}\right) \cong{ }^{H} \tilde{\mathcal{O}}(V) / \sum_{j}^{H} \tilde{\mathcal{O}}\left(\hat{V}_{j}\right)$, where $H^{n}\left(W, W^{\prime} ;{ }^{H} \tilde{\mathcal{O}}\right)$ is the relative cohomology of covering. Therefore, combining with Proposition 3.12 we have the isomorphism $H_{\mathbb{D} n}^{n}\left(\mathbb{Q}^{n},{ }^{H} \tilde{\mathcal{O}}\right) \cong L\left(\mathcal{Q}\left(\mathbb{D}^{n}\right), H\right)$, which provides Theorem $3.8^{\prime}$.

Proposition 3.13. Let $K=K_{1} \times \ldots \times K_{n}$, each $K_{j}$ being a compact subset of $\mathbb{D}$. $\kappa(\Phi)$ for every $\Phi \in L(\mathcal{Q}(K), H)$ is an element of $\sum_{j}{ }^{H} \mathscr{O}\left(X_{j}\right)$, where $X_{j}=\hat{V}_{j} \cap\left\{z_{j} \notin K_{j}\right\}$.

Proof. $\phi(z)=\Phi\left(h_{z}\right)$ is analytic if $z_{j} \notin K_{j}$ for all $j$.

If $U$ is an open set in $\mathbb{Q}^{n}$ we denote by $\varrho_{U}$ the restriction map from

$$
{ }^{H} \tilde{\mathcal{O}}(V) / \sum_{j}{ }^{H} \tilde{\mathcal{O}}\left(\hat{V}_{j}\right) \quad \text { to }{ }^{H} \mathscr{R}(U)={ }^{H} \tilde{\mathcal{O}}(V \cap U) / \sum_{j}{ }^{H} \tilde{\mathcal{O}}\left(\hat{V}_{j} \cap U\right) \text {. }
$$

By means of the map $\varrho_{U}$ we can define the support of an element $\phi$ of ${ }^{H} \tilde{\mathcal{O}}(V) / \sum_{j}{ }^{H} \tilde{\mathcal{O}}\left(\hat{V}_{j}\right)$ as the smallest closed set $S$ such that $\varrho_{U}(\phi)=0$ for any open subset $U \subset S^{c}$. 
If $U \supset U^{\prime}$ we further denote by $\varrho_{U^{\prime}}^{U}$ the restriction map from ${ }^{H} \mathscr{R}(U)$ to ${ }^{H} \mathscr{R}\left(U^{\prime}\right)$. It is easy to see the following properties of $\varrho_{U}^{U}: \varrho_{U}^{U}$ is identity, and if $U \supset U^{\prime} \supset U^{\prime \prime}$, then $\varrho_{U^{\prime \prime}}^{U^{\prime}} \varrho_{U^{\prime}}^{U}=\varrho_{U^{\prime \prime}}^{U}$. Hence $\left\{{ }^{H} \mathscr{R}(U), \varrho_{U^{\prime}}^{U}\right\}$ forms a presheaf (over $\mathbb{Q}^{n}$ ). Ito and Nagamachi [7] have verified that $\left\{{ }^{H} \mathscr{R}(U), \varrho_{U^{\prime}}^{U}\right\}$ really is a flabby sheaf, that is

(i) If for any $z \in U$ there exists a neighbourhood $V(z)$ of $z$ such that $\varrho_{V(z)}^{U}(\phi)=0$, then $\phi=0$.

(ii) Let $\left\{V_{\sigma}\right\}$ be an open covering of $U$. If $\phi_{\sigma} \in^{H} \mathscr{R}\left(V_{\sigma}\right)$ satisfies $\varrho_{V_{\sigma}}^{V_{\sigma}} V_{\tau}\left(\phi_{\sigma}\right)=$ $\varrho_{V_{\sigma} \cap V_{\tau}}^{V_{\tau}}\left(\phi_{\tau}\right)$ for $V_{\sigma} \cap V_{\tau} \neq \emptyset$, then there exists $\phi \in{ }^{H} \mathscr{R}(U)$ such that $\varrho_{V_{\sigma}}^{U}(\phi)=\phi_{\sigma}$.

(iii) (Flabbiness) $\varrho_{U}$ is surjective.

We denote by ${ }^{H} \mathscr{R}$ the sheaf $\left\{{ }^{H} \mathscr{R}(U), \varrho_{U}^{U}\right\}$ over $\mathbb{Q}^{n}$. But the support of ${ }^{H} \mathscr{R}$ is $\mathbb{D}^{n}$ so that ${ }^{H} \mathscr{R}$ may be considered as a sheaf over $\mathbb{D}^{n}$. We call ${ }^{H} \mathscr{R}$ the sheaf of $H$-valued Fourier hyperfunctions.

Proposition 3.14. Let $K$ be a compact set of $\mathbb{D}^{n}$ and $\Phi$ an element of $L(Q(K), H)$, then the support of $\kappa(\Phi)$ is contained in $K$.

For the proof of this proposition we need

Lemma 3.15. Let $K$ be a compact set in $\mathbb{D}^{n}$ and let $\left\{K_{\sigma}\right\}$ be a finite compact covering of $K$. Then every element $\Phi$ of $L(\mathcal{Q}(K), H)$ can be decomposed into $\Phi=\sum_{\sigma} \Phi_{\sigma}$ with $\Phi_{\sigma} \in L\left(Q\left(K_{\sigma}\right), H\right)$.

Proof. First we prove the lemma for the scalar-valued case. Since the natural injection $\mathscr{Q}(K) \rightarrow \oplus_{\sigma} \mathcal{Q}\left(K \cap K_{\sigma}\right)$ is of closed range, the dual map $\oplus_{\sigma}\left(\mathcal{Q}\left(K \cap K_{\sigma}\right)\right)^{\prime} \rightarrow$ $(\mathscr{Q}(K))^{\prime}$ is surjective. In the vector-valued case the lemma follows from the isomorphisms, $L(\mathcal{Q}(K), H) \cong(\mathscr{Q}(K))^{\prime} \hat{\otimes} H$ and $L\left(\mathscr{Q}\left(K_{\sigma}\right), H\right) \cong\left(\mathscr{Q}\left(K_{\sigma}\right)\right)^{\prime} \hat{\otimes} H$, which are obtained by Proposition 50.6 of Treves [13], because $\mathcal{Q}(K)$ and $\mathcal{Q}\left(K_{\sigma}\right)$ are both DFS-spaces and so they are complete and barreled [7].

We now return to the proof of Proposition 3.14. If $z \notin K$ there exist an open neighbourhood $U$ of $z$ and a finite compact covering $\left\{K_{\sigma}\right\}$ of $K$ such that $K_{\sigma} \cap U=\emptyset, K_{\sigma}=K_{\sigma 1} \times \ldots \times K_{\sigma n}$ and $U=U_{1} \times \ldots \times U_{n}$. Then we have $\Phi=\sum_{\sigma} \Phi_{\sigma}$, $\Phi_{\sigma} \in L\left(\mathcal{Q}\left(K_{\sigma}\right), H\right)$, by the lemma and $\varrho_{U}\left(\kappa\left(\Phi_{\sigma}\right)\right)=0$ by Proposition 3.13. The proof of Proposition 3.14 is thus completed.

Remark. Lemma 3.15 is a direct consequence of the flabbiness of the sheaf ${ }^{H} \mathscr{R}$.

Definition 3.16. For $\Phi \in L\left(Q\left(\mathbb{D}^{n}\right), H\right)$ we define its Fourier transform $\mathscr{F} \Phi$ by the formula $(\mathscr{F} \Phi)(\mathscr{F} f)=\Phi(f)$, where

$$
(\mathscr{F} f)(p)=\int e^{i(p, x)} f(x) d x
$$

for $f \in \mathcal{Q}\left(\mathbb{D}^{n}\right)$.

Since $\mathscr{F}$ yields a topological isomorphism of $\mathcal{Q}\left(\mathbb{D}^{n}\right)$ (see $\S \S 2.3$ and 2.4 ), the definition above is well defined. The sheaf ${ }^{H} \mathscr{B}$ of $\tilde{H}$-valued hyperfunctions over $\mathbb{R}^{n}$ coincides with the restriction of the sheaf ${ }^{H} \mathscr{R}$ to $\mathbb{R}^{n}$. Hence, because of the flabbiness of the sheaf ${ }^{H} \mathscr{R}$, any $H$-valued Fourier hyperfunction on $\mathbb{R}^{n}$ can be extended to an $H$-valued hyperfunction on $\mathbb{I D}^{n}$ and we can consider its Fourier transformation.

Proposition 3.17 (Paley-Wiener theorem). Let $\Gamma$ be a closed and strictly convex cone in $\mathbb{R}^{n}$ with its vectex at the origin such that $\Gamma \subset\{x ;(x, e)>0\} \cup\{0\}$ for a unit 
vector $e$ in $\mathbb{R}^{n}$. We denote by $K$ the closure of $\Gamma$ in $\mathbb{D}^{n}$, and by $\Gamma^{0}$ the polar set of $\Gamma$, namely $\Gamma^{0}=\{x ;(x, \xi) \geqq 0, \xi \in \Gamma\}$. Then $\Phi$ is an element of $L(\mathcal{Q}(K), H)$ if and only if $f(x)=\Phi\left(e^{i(\cdot, x)}\right)$ is holomorphic in $\mathbb{R}^{n} \times i\left(\Gamma^{0}\right)^{i}$, where $\left(\Gamma^{0}\right)^{i}$ is the set of interior points of $\Gamma^{0}$, and satisfies the condition that for every $\Gamma^{\prime} \Subset \Gamma^{0}$ and $\varepsilon>0$

$$
\left\|\Phi\left(e^{i(\cdot, \zeta)}\right)\right\| \leqq C_{\varepsilon, \Gamma^{\prime}} \exp \left\{\varepsilon|\operatorname{Re} \zeta|+\chi_{\Gamma, \varepsilon}(\operatorname{Im} \zeta)\right\}
$$

where $\zeta \in \mathbb{R}^{n} \times i \Gamma^{\prime}$ and $\chi_{\Gamma, \varepsilon}(\eta)=\sup _{x \in(\Gamma-\varepsilon e)}\{-(x, \eta)+\varepsilon|x|\}$.

Proof. See Ito and Nagamachi [7].

At the end of this section we mention the sheaf $\mathscr{C}$ and Sato's fundamental principle. Let $M$ be an oriented real analytic manifold of dimension $n$, and let $T^{*} M$ be the cotangential bundle over $M$, and finally let $S^{*} M$ be the cosphere bundle $S^{*} M=\left(T^{*} M-M\right) / \mathbb{R}^{+}$. When $M=\mathbb{R}^{n}$, the cosphere bundle $S^{*} M$ is represented by $\mathbb{R}^{n} \times \mathbb{S}^{n-1}$.

We can construct a sheaf $\mathscr{C}$ over $S^{*} M$ and a sheaf homomorphism $\beta$ from the sheaf $\mathscr{B}$ of germs of hyperfunctions over $M$ to the direct image $\pi_{*} \mathscr{C}$ of the sheaf $\mathscr{C}$ by the projection $\pi: S^{*} M \rightarrow M$ in such a way that the following proposition is true.

Proposition 3.18. The sequence of sheaves over $M$

$$
0 \rightarrow \mathscr{A} \stackrel{\alpha}{\rightarrow} \mathscr{B} \stackrel{\beta}{\rightarrow} \pi_{*} \mathscr{C} \rightarrow 0
$$

is exact. Here $\mathscr{A}$ is the sheaf of germs of real analytic functions over $M$ and $\alpha: \mathscr{A} \rightarrow \mathscr{B}$ is the canonical injection.

For the construction of $\mathscr{C}$ we refer to a paper of Sato, Kawai, and Kashiwara [14].

For a hyperfunction $\phi \in \mathscr{B}(\Omega)$ on an open set $\Omega \subset \mathbb{R}^{n}, \beta \phi$ defines a section of the sheaf $\mathscr{C}$ over $\pi^{-1}(\Omega)=\Omega \times \mathbb{S}^{n-1}$ and we can consider the support of $\beta \phi$. We call it the singularity spectrum of $\phi$ and denote it by S.S. $\phi$.

Proposition 3.19. Let $\phi$ be a hyperfunction of $\mathscr{B}(\Omega)$ and $\left\{\Gamma_{k}\right\}_{k=1}^{m}$ be open convex cones. Then the following two conditions are equivalent:

(i) $\phi$ can be represented by boundary values of holomorphic functions $f_{k}(z)$ defined on each $\Omega \times i \Gamma_{k}, 1 \leqq k \leqq m$.

(ii) S.S. $\phi \subset \bigcup_{k=1}^{m} \Gamma_{k}^{0}$.

Proof. See Morimoto [15].

Proposition 3.20. (Sato's fundamental principle). Let $P(x, D)$ be a linear partial differential operator of order $m$ whose coefficients are real analytic on an open set $\Omega \subset \mathbb{R}^{n}$ and let $P_{m}(x, \xi)$ be its principal symbol: Write $P(x, D)=\sum_{|\alpha|=m} a_{\alpha}(x) D^{\alpha}+$ $\sum_{|\beta| \leqq m-1} b_{\beta}(x) D^{\beta}$, then the principal symbol is defined by $P_{m}(x, \xi)=\sum_{|\alpha|=m}^{|\alpha|=m} a_{\alpha}(x) \xi^{\alpha}$. If $\phi \in \mathscr{B}(\Omega)$ satisfies $P(x, D) \phi=0$, then S.S. $\phi \subset\left\{(x, \xi) \in \Omega \times \mathbb{S}^{n-1} ; P_{m}(x, \xi)=0\right\}$.

Proof. See Sato [16]. 


\section{§4. Axioms for Hyperfunction Quantum Field Theory}

In extending the usual Wightman framework of the axiomatic quantum field theory our greatest concern is how to formulate the locality axiom for extended theory. The strict localizability of fields $A(f)$ connects mathematically with the fact that $f(x)$ belongs to a function space which contains $C^{\infty}$ functions with compact support. This requirement is satisfied by Wightman fields and Jaffe fields, because these fields are constructed on the basis of the spaces $\mathscr{S}$ and $\mathscr{C}_{\omega}$, respectively. The advantage of the symmetry between configuration and momentum spaces is given to Wightman tempered fields, while it is lost in the Jaffe field theory, since the space $\mathscr{S}$ is topologically invariant under the Fourier transformation, but the space $\mathscr{C}_{\omega}$ is not. Constantinescu [3] used the space $\mathscr{S}^{1}$ to construct local but nonstrictly localizable fields. He proved that the theory can be formulated so that Wightman functions in configuration space are still boundary values of analytic functions in the forward tube, but can grow arbitrarily fast near the light cone in contrast to the case of tempered fields where they are only of polynomial growth.

Here we wish to formulate axioms for Fourier hyperfunction fields. Since the test function space $\mathcal{Q}(\mathbb{D})$ of Fourier hyperfunctions no longer contains any function of compact support, we are obliged to modify the axiom of locality in an appropriate way. Instead, it should be remarked that the symmetry between configuration and momentum spaces is recovered in the hyperfunction quantum field theory. Most of the axioms can be stated in parallel with Wightman's axioms for tempered fields, but for completeness we write down them mutatis mutandis following Jost [17] (see also [1]).

The Lorentz-invariant inner product is introduced in $\mathbb{R}^{4}$ by writting $x \cdot y=$ $x^{0} y^{0}-\boldsymbol{x} \cdot \boldsymbol{y}$ for two four-vectors $x=\left(x^{0}, \boldsymbol{x}\right)$ and $y=\left(y^{0}, \boldsymbol{y}\right)$, where $\boldsymbol{x}$ and $\boldsymbol{y}$ are threedimensional spatial vectors. Write also $x^{2}=x \cdot x$ to make short the length of expressions. In the same spirit we shall use the notation $\underline{x}_{n}$ to signify a set of $n$ four-vectors $\left(x_{1}, \ldots, x_{n}\right)$ and write $d \underline{x}_{n}$ instead of $d x_{1} \ldots d x_{n}$.

Zeroth Axiom. The space of states is a separable Hilbert space $H$ over the complex number $\mathbb{C}$. For $\Phi, \Psi \in H$ the inner product in $H$ will be denoted by $(\Phi, \Psi)$.

First Axiom. $Q\left(\mathbb{D}^{4}\right)$ is linearly mapped into linear operators $\{A(f)\}$ over $H$. $A(f)$ is defined on a dense subset $D$ of $H$, independent of $f \in \mathcal{Q}\left(\mathbb{D}^{4}\right) .(\Phi, A(f) \Psi)$ for $\Phi, \Psi \in D$ is a Fourier hyperfunction and $(\Phi, A(f) \Psi)=(A(\tilde{f}) \tilde{\Phi}, \Psi)$. We require that $A(f) D \subset D$.

Second Axiom. A unitary representation $U(a, \Lambda)$ of the restricted Poincare group exists and satisfies

$$
U(a, \Lambda) A(f) U^{-1}(a, \Lambda)=A\left(f_{\{a, \Lambda\}}\right)
$$

and $U(a, \Lambda) D=D$, where $f_{\{a, \Lambda\}}(x)=f\left(\Lambda^{-1}(x-a)\right)$.

Third Axiom. The spectrum of the energy-momentum operator $P$ is contained in $\bar{V}_{+}$and there is an invariant state $\Omega$, corresponding to the vacuum, such that $U(a, \Lambda) \Omega=\Omega$. Here $V_{+}=\left\{\left(p^{0}, \boldsymbol{p}\right) ; p^{0}>0, p^{2}>0\right\}$ and $\bar{V}_{+}$is the closure of $V_{+}$in $\mathbb{D}^{4}$. 
By the first axiom $\left(\Phi, A\left(f_{1}\right) \ldots A\left(f_{n}\right) \Psi\right)$ for $\Phi, \Psi \in D$ is a separately continuous multilinear form on $\left[Q \mathcal{Q}\left(\mathbb{D}^{4}\right)\right]^{n}$. Proposition 3.6 says that $\left(\Phi, A\left(f_{1}\right) \ldots A\left(f_{n}\right) \Psi\right)$ uniquely determines a Fourier hyperfunction belonging to $\left(\mathscr{Q}\left(\mathbb{D}^{4 n}\right)\right)^{\prime}$ which we denote by $\left(\Phi, A\left(x_{1}\right) \ldots A\left(x_{n}\right) \Psi\right)$ in the sense that formally

$$
\begin{aligned}
(\Phi, & \left.A\left(f_{1}\right) \ldots A\left(f_{n}\right) \Psi\right) \\
\quad & \int\left(\Phi, A\left(x_{1}\right) \ldots A\left(x_{n}\right) \Psi\right) f_{1}\left(x_{1}\right) \ldots f_{n}\left(x_{n}\right) d \underline{x}_{n} .
\end{aligned}
$$

More generally we write

$$
F(f)=\int F\left(\underline{x}_{n}\right) f\left(\underline{x}_{n}\right) d \underline{x}_{n}
$$

for $F \in \mathbb{R}\left(\mathbb{D}^{4 n}\right)$ and $f \in \mathcal{Q}\left(\mathbb{D}^{4 n}\right)$.

Next for $g\left(\underline{x}_{n}\right)=\tilde{f_{1}}\left(x_{1}\right) \ldots f_{n}\left(x_{n}\right), f_{j} \in \mathcal{Q}\left(\mathbb{D}^{4}\right)$, and $\Phi \in D$ we define an $H$-valued functional $\Phi(g)=A\left(f_{1}\right) \ldots A\left(f_{n}\right) \Phi$ and extend this definition to $\bigotimes_{n} \mathcal{O}\left(\mathbb{D}^{4}\right)$ by linearity. By Proposition 3.5, for any $f \in \mathcal{Q}\left(\mathbb{D}^{4 n}\right)$ there exists a net $\left\{g_{v} ; g_{v} \in \underset{n}{\otimes} \mathcal{Q}\left(\mathbb{D}^{4}\right)\right\}$ such that $g_{v} \rightarrow f$ as $v \rightarrow \infty$. Therefore $\left\|\Phi_{n}\left(g_{v}\right)-\Phi_{n}\left(g_{\mu}\right)\right\|^{2} \rightarrow 0$ as $v, \mu \rightarrow \infty$. Thus $\Phi_{n}\left(g_{v}\right)$ converges and tends to a vector $\Phi_{n}(f)$ and evidently $\Phi_{n}(f)$ is a continuous linear mapping from $\mathcal{Q}\left(\mathbb{D}^{4 n}\right)$ to $H$, i.e., $\Phi_{n}(f) \in L\left(\mathcal{Q}\left(\mathbb{D}^{4 n}\right), H\right)$. By Theorem $3.8^{\prime} \Phi_{n}(f)$ is an $H$-valued Fourier hyperfunction. We denote it symbolically by $A\left(x_{1}\right) \ldots A\left(x_{n}\right) \Phi$. On the other hand, $\Phi_{n}(f)$ defines a linear operator on $D$ which maps $\Phi$ to $\Phi_{n}(f)$. That operator will be denoted by $\left\langle A^{n}, f\right\rangle$ and written formally as

$$
\left\langle A^{n}, f\right\rangle=\int A\left(x_{1}\right) \ldots A\left(x_{n}\right) f\left(\underline{x}_{n}\right) d \underline{x}_{n} .
$$

The axiom of local commutativity then is formulated as follows:

Fourth Axiom. If $x_{1}$ and $x_{2}$ are spacelike separated, then $A\left(x_{1}\right) A\left(x_{2}\right) \Phi=$ $A\left(x_{2}\right) A\left(x_{1}\right) \Phi$ for every $\Phi \in \mathrm{D}$.

By Theorem $3.8^{\prime}$ we can restate this axiom by saying that $A\left(x_{1}\right) A\left(x_{2}\right) \Phi-$ $A\left(x_{2}\right) A\left(x_{1}\right) \Phi \in L(\mathcal{Q}(K), H)$ for every $\Phi \in D$, where $K$ is the closure of $\left\{\left(x_{1}, x_{2}\right) \in \mathbb{R}^{8}\right.$; $\left.\left(x_{1}-x_{2}\right)^{2}>0\right\}$ in $D^{8}$. More precisely we should formulate the locality axiom by means of the support concept of $H$-valued Fourier hyperfunctions. Thus, let $U_{1}$ and $U_{2}$ be arbitrary open sets in $\mathbb{R}^{4}$ which are spacelike separated to each other, namely let $\left(x_{1}-x_{2}\right)^{2}<0$ for every $x_{1} \in U_{1}$ and $x_{2} \in U_{2}$. Consider $\Phi_{2}(f)=$ $A\left(f_{1}\right) A\left(f_{2}\right) \Phi \in L\left(Q\left(\mathbb{D}^{8}\right), H\right)$. The locality axiom should read

$$
\varrho_{U_{12}}\left(\kappa\left(\Phi_{2}\right)\right)=\varrho_{U_{21}}\left(\kappa\left(\Phi_{2}\right)\right) \text {, }
$$

where $U_{12}=U_{1} \times U_{2}$ and $U_{21}=U_{2} \times U_{1}$; the mapping $\kappa$ and the restriction $\varrho$ are defined in the previous section.

Fifth Axiom. Let $P(A)$ be an algebra of polynomials in the operators $A(f)$, $f \in \mathscr{Q}\left(\mathbb{D}^{4}\right)$, then $P(A) \Omega$ is dense in $H$.

\section{$\S$ 5. Properties of Vacuum Expectation Values}

Let $\Omega$ be the vacuum vector. The vacuum expectation value $\left(\Omega, A\left(x_{1}\right) \ldots A\left(x_{n}\right) \Omega\right)$ is a Fourier hyperfunction which we denote by $\mathfrak{B B}_{n}\left(\underline{x}_{n}\right)$, where $\underline{x}_{n}=\left(x_{1}, x_{2}, \ldots, x_{n}\right)$ as before. When the order of the vectors is reversed we shall use the notation ${ }_{n} \underline{x}:{ }_{n} \underline{x}=\left(x_{n}, x_{n-1}, \ldots, x_{1}\right)$. 
Proposition 5.1 (Positive definiteness). For any sequence $\left\{\phi_{k}\right\}$ of test functions $\phi_{k} \in \mathcal{Q}\left(\mathbb{D}^{4 k}\right)$ with $\phi_{k}=0$ except for a finite number of $k$ 's, the vacuum expectation values satisfy the inequality

$$
\sum_{k, l=0}^{\infty} \int \bar{\phi}_{k}\left(\underline{x}_{k}\right) \mathfrak{B}_{k+l}\left({ }_{k} \underline{x}, \underline{y}_{l}\right) \phi_{l}\left(\underline{y}_{l}\right) d \underline{x}_{k} d \underline{y}_{l} \geqq 0 .
$$

Proof. Let $\Omega_{n}\left(\phi_{n}\right)=\left\langle A^{n}, \phi_{n}\right\rangle \Omega$. The inequality (5.1) is equivalent to

$$
\left\|\sum_{n=0}^{\infty} \Omega_{n}\left(\phi_{n}\right)\right\|^{2} \geqq 0
$$

which follows from the zeroth axiom.

Proposition 5.2 (Hermiticity).

$$
\mathfrak{W}_{n}\left(\underline{x}_{n}\right)=\overline{\mathfrak{W} \mathfrak{B}_{n}(n \underline{x})} \text {. }
$$

Proposition 5.3 (Relativistic invariance).

$$
\mathfrak{W}_{n}\left(\Lambda \underline{x}_{n}+a\right)=\mathfrak{W}_{n}\left(\underline{x}_{n}\right),
$$

where $\Lambda \underline{x}_{n}+a=\left(\Lambda x_{1}+a, \ldots, \Lambda x_{n}+a\right)$.

These two propositions are direct consequences of the first and the second axioms, respectively.

Proposition 5.4 (Spectral condition). There are Fourier hyperfunctions $W_{n-1}\left(\xi_{n-1}\right)$ depending on the relative coordinates $\xi_{j}=x_{j+1}-x_{j}, j=1,2, \ldots, n-1$, which are related $\mathfrak{W}_{n}$ by $\mathfrak{W}_{n}\left(\underline{x}_{n}\right)=W_{n-1}\left(\xi_{n-1}\right)$. The Fourier transforms of $\mathfrak{W}_{n}$ and $W_{n-1}$ are Fourier hyperfunctions defined by

$$
\tilde{\mathfrak{W}}_{n}\left(\underline{p}_{n}\right)=(2 \pi)^{-4 n} \int \exp \left\{-i \sum_{j=1}^{n} p_{j} \cdot x_{j}\right\} \mathfrak{W}_{n}\left(\underline{x}_{n}\right) d \underline{x}_{n}
$$

and

$$
\tilde{W}_{n-1}\left(q_{n-1}\right)=(2 \pi)^{-4(n-1)} \int \exp \left\{-i \sum_{j=1}^{n-1} q_{j} \cdot \xi_{j}\right\} W_{n-1}\left(\xi_{n-1}\right) d \xi_{n-1} .
$$

These are related by

$$
\tilde{\mathfrak{B}}_{n}\left(\underline{p}_{n}\right)=\delta\left(\sum_{j=1}^{n} p_{j}\right) \tilde{W}_{n-1}\left(p_{2}+\ldots+p_{n}, p_{3}+\ldots+p_{n}, \ldots, p_{n}\right)
$$

and the support of $\tilde{W}_{n-1}\left(\underline{q}_{n-1}\right)$ is contained in $\bar{V}_{+}^{n-1}$.

Proof. We make a linear transformation $\underline{x}_{n} \rightarrow\left(\xi_{0}, \underline{\xi}_{n-1}\right)$ with $\xi_{0}=x_{1}$ and define

$$
\begin{aligned}
\mathfrak{W}_{n}\left(\underline{x}_{n}\right) & =\mathfrak{W}_{n}\left(\xi_{0}, \xi_{0}+\xi_{1}, \ldots, \xi_{0}+\ldots+\xi_{n-1}\right) \\
& \equiv \mathfrak{W}_{n}\left(\xi_{0}, \underline{\xi}_{n-1}\right) .
\end{aligned}
$$

Since $\mathfrak{W}_{n}$ is translation-invariant it obeys the equation $\left(\partial / \partial \xi_{0}^{\mu}\right) \mathfrak{W B}_{n}^{\prime}\left(\xi_{0}, \underline{\xi}_{n-1}\right)=0$, $\mu=0,1,2,3$. By Sato's fundamental principle on the singularity spectrum of hyperfunctions (Proposition 3.20), $\mathfrak{W}_{n}^{\prime}\left(\xi_{0}, \underline{\xi}_{n-1}\right)$ is real analytic in $\xi_{0}[14]$. Let us define a Fourier hyperfunction with $n-1$ arguments

$$
W_{n-1}\left(\underline{\xi}_{n-1}\right)=\mathfrak{W} \mathfrak{B}_{n}^{\prime}\left(0, \underline{\xi}_{n-1}\right)=\mathfrak{W}_{n}^{\prime}\left(\xi_{0}, \underline{\xi}_{n-1}\right),
$$

then $\mathfrak{B}_{n}\left(\underline{x}_{n}\right)=W_{n-1}\left(\underline{\xi}_{n-1}\right)$ as required. 
Next it is easy to see (5.6): we have

$$
\begin{aligned}
\tilde{\mathfrak{W}}_{n}\left(\underline{p}_{n}\right)= & (2 \pi)^{-4 n} \int \exp \left\{-i \sum_{j=1}^{n} p_{j} \cdot x_{j}\right\} \mathfrak{W}_{n}\left(\underline{x}_{n}\right) d \underline{x}_{n} \\
= & (2 \pi)^{-4 n} \int \exp \left\{-i\left[\left(p_{2}+\ldots+p_{n}\right) \cdot \xi_{1}+\ldots+p_{n} \cdot \xi_{n-1}\right]\right\} \\
& \cdot \exp \left\{-i\left(\sum_{j=1}^{n} p_{j}\right) \cdot \xi_{0}\right\} W_{n-1}\left(\underline{\xi}_{n-1}\right) d \xi_{0} d \underline{\xi}_{n-1} \\
= & \delta\left(\sum_{j=1}^{n} p_{j}\right) \tilde{W}_{n-1}\left(p_{2}+\ldots+p_{n}, p_{3}+\ldots+p_{n}, \ldots, p_{n}\right) .
\end{aligned}
$$

The last statement of Proposition 5.4 can be proved as follows: $\Omega_{n}(\phi)=\left\langle A^{n}, \phi\right\rangle \Omega$ is an $H$-valued Fourier hyperfunction. When the translation $U(a, 1)$ is applied to $\Omega_{n}(\phi)$ we have by the second axiom

$$
\begin{aligned}
U(a, 1) \Omega_{n}(\phi) & =\Omega_{n}\left(\phi_{\{a, 1\}}\right) \\
& =\tilde{\Omega}_{n}\left(\tilde{\phi} \exp \left\{i\left(\sum_{j=1}^{n} p_{j}\right) \cdot a\right\}\right),
\end{aligned}
$$

where $\tilde{\phi}$ is the Fourier transform of $\phi$. Consider the spectral resolution

$$
U(a, 1)=\int e^{i p \cdot a} d E(p)
$$

and the integral

$$
\int \chi(p) d E(p) \Omega_{n}(\phi)=\tilde{\Omega}_{n}\left(\chi\left(p_{1}+\ldots+p_{n}\right) \tilde{\phi}\right)
$$

for any $\chi(p) \in \mathcal{Q}\left(\mathbb{D}^{4}\right)$. The mapping $\chi \rightarrow \tilde{\Omega}_{n}\left(\chi\left(p_{1}+\ldots+p_{n}\right) \tilde{\phi}\right)$ defined by the integral (5.8) is an element of $L\left(\mathcal{Q}\left(\mathbb{D}^{4}\right), H\right)$, but since $\operatorname{supp}(d E) \subset \bar{V}_{+}$by the third axiom it really belongs to $L\left(\mathcal{Q}\left(\bar{V}_{+}\right), H\right)$. Let $S_{0}$ be the closure of $\left\{p_{n} ; p_{1}+\ldots+p_{n} \in \bar{V}_{+}\right\}$in $\mathrm{D}^{4 n}$, then what we have just ascertained shows that $\tilde{\Omega}_{n}\left(\underline{p}_{n}\right) \in L\left(\mathcal{\sim}\left(S_{0}\right), H\right)$. It is readily seen that $\left\langle A^{k}, \psi\right\rangle \tilde{\Omega}_{n-k}(\tilde{\phi})=\tilde{\Omega}_{n}(\tilde{\psi} \otimes \tilde{\phi})$ and $\left\langle A^{k}, \psi\right\rangle \int \chi(p) d E(p) \tilde{\Omega}_{n-k}(\tilde{\phi})=$ $\tilde{\Omega}_{n}\left(\tilde{\psi} \otimes \chi\left(p_{k+1}+\ldots+p_{n}\right) \tilde{\phi}\right)$ for $1 \leqq k \leqq n-1$. Let us similarly define $S_{k}$ as the closure of $\left\{\underline{p}_{n} ; p_{k+1}+\ldots+p_{n} \in \bar{V}_{+}\right\}$in $\mathbb{D}^{4 n}$. Then $\tilde{\Omega}_{n}\left(\underline{p}_{n}\right) \in L\left(\mathcal{Q}\left(\bigcap_{k=0}^{n-1} S_{k}\right), H\right)$. To put this in another way let us write $q_{k}=p_{k+1}+\ldots+p_{n}, 0 \leqq k \leqq n-1$, and

$$
\begin{aligned}
\tilde{\Omega}_{n}\left(\underline{p}_{n}\right) & =\tilde{\Omega}_{n}\left(q_{0}-q_{1}, q_{1}-q_{2}, \ldots, q_{n-1}\right) \\
& \equiv \tilde{Z}_{n}\left(q_{0}, \underline{q}_{n-1}\right),
\end{aligned}
$$

then we obtain $\tilde{Z}_{n}\left(q_{0}, \underline{q}_{n-1}\right) \in L\left(\mathcal{\sim}_{(}\left(\bar{V}_{+}^{n}\right), H\right)$. Since

$$
\begin{aligned}
\tilde{\mathfrak{W}}_{n}\left(\underline{p}_{n}\right) & =\left(\Omega, \tilde{\Omega}_{n}\left(\underline{p}_{n}\right)\right)=\left(\Omega, \tilde{Z}_{n}\left(q_{0}, \underline{q}_{n-1}\right)\right) \\
& =\delta\left(q_{0}\right) \tilde{W}_{n-1}\left(\underline{q}_{n-1}\right),
\end{aligned}
$$

$\tilde{W}_{n-1}\left(q_{-1}\right)$ is a Fourier hyperfunction whose support is contained in $\bar{V}_{+}^{n-1}$. This completes the proof of Proposition 5.4. 


\section{§6. Reconstruction}

Theorem 6.1. To a given set of Wightman Fourier hyperfunctions satisfying the conditions expressed in Propositions 5.1-5.4 in \$ 5, there corresponds uniquely a neutral scalar field $A(f)$ which obeys all the axioms of hyperfunction fields and has the Fourier hyperfunctions as vacuum expectation values.

Proof. We can construct a Hilbert space $H$, the field operator $A(f)$ and the unitary representation $U(a, \Lambda)$ of the restricted Poincare group in the same way as tempered fields. Therefore we discuss only causality and spectral condition.

(a) Causality

$$
\begin{aligned}
& \left(A\left(x_{1}\right) A\left(x_{2}\right) A\left(u_{1}\right) \ldots A\left(u_{n}\right) \Omega-A\left(x_{2}\right) A\left(x_{1}\right) A\left(u_{1}\right) \ldots A\left(u_{n}\right) \Omega\right. \\
& \left.A\left(y_{1}\right) A\left(y_{2}\right) A\left(v_{1}\right) \ldots A\left(v_{n}\right) \Omega-A\left(y_{2}\right) A\left(y_{1}\right) A\left(v_{1}\right) \ldots A\left(v_{n}\right) \Omega\right) \\
& \quad=\mathfrak{W}_{2 n+2}\left({ }_{n} \underline{u}, x_{2}, x_{1}, y_{1}, y_{2}, \underline{v}_{n}\right)-\mathfrak{B}_{2 n+2}\left({ }_{n} \underline{u}, x_{1}, x_{2}, y_{1}, y_{2}, \underline{v}_{n}\right) \\
& \quad+\mathfrak{W}_{2 n+2}\left({ }_{n} \underline{u}, x_{2}, x_{1}, y_{2}, y_{1}, \underline{v}_{n}\right)-\mathfrak{W}_{2 n+2}\left({ }_{n} \underline{u}, x_{1}, x_{2}, y_{2}, y_{1}, \underline{v}_{n}\right) \\
& \quad=0
\end{aligned}
$$

if $\left(x_{1}-x_{2}\right)^{2}<0$ or $\left(y_{1}-y_{2}\right)^{2}<0$, i.e., if $\left(x_{1}, x_{2}, y_{1}, y_{2}\right) \in\left(K^{c} \times \mathbb{D}^{4}\right) \cup\left(\mathbb{D}^{4} \times K^{c}\right)$, where $K$ is the closure of $\left\{\left(x_{1}, x_{2}\right) ;\left(x_{1}-x_{2}\right)^{2}>0\right\}$ in $\mathbb{D}^{8}$ and $K^{c}$ is its complement. Thus the support of the Fourier hyperfunction above is contained in

$$
\left(K^{c} \times \mathbb{D}^{4} \cup \mathbb{D}^{4} \times K^{c}\right)^{c}=K \times K .
$$

Therefore, if $\Phi$ is a linear combination of vectors of the form $A\left(\phi_{1}\right) \ldots A\left(\phi_{n}\right) \Omega$, then $A\left(x_{1}\right) A\left(x_{2}\right) \Phi-A\left(x_{2}\right) A\left(x_{1}\right) \Phi \in L(Q(K), H)$, that is $A\left(x_{1}\right) A\left(x_{2}\right) \Phi=A\left(x_{2}\right) A\left(x_{1}\right) \Phi$ if $x_{1}$ and $x_{2}$ are spacelike separated.

\section{(b) Spectral Condition}

Once $H$-valued Fourier hyperfunctions $\tilde{\Omega}_{n}\left(\underline{p}_{n}\right)$ have been constructed, it follows from the relations

$$
\begin{aligned}
\left(\tilde{\Omega}_{n}\left(\underline{p}_{n}\right), \tilde{\Omega}_{n}\left(\underline{q}_{n}\right)\right) & =\overline{\left(\tilde{\Omega}_{n}\left(\underline{q}_{n}\right), \tilde{\Omega}_{n}\left(\underline{p}_{n}\right)\right)} \\
& =\tilde{\mathfrak{W}}_{2 n}\left({ }_{n} \underline{p}, \underline{q}_{n}\right)=\overline{\tilde{\mathfrak{B}}_{2 n}\left({ }_{n} \underline{q}, \underline{p}_{n}\right)}
\end{aligned}
$$

and the support property of Wightman Fourier hyperfunctions that the support of $\tilde{\mathfrak{B}}_{2 n}\left({ }_{n} p, q_{n}\right)$ is contained in $S_{0} \times S_{0}$, where $S_{0}$ is the closed subset of $\mathbb{D}^{4 n}$ defined in the preceding section. Therefore $\tilde{\Omega}_{n}\left(p_{n}\right)$ is an element of $L\left(\mathcal{Q}\left(S_{0}\right), H\right)$. For any $\chi(p) \in \mathcal{Q}\left(\bar{V}_{+}\right) \cap \mathscr{S}$ we have $\int \chi(p) d E(p) \tilde{\Omega}_{n}(\tilde{\phi})=\tilde{\Omega}_{n}\left(\chi\left(p_{1}+\ldots+p_{n}\right) \tilde{\phi}\right)$, where $E(p)$ is the resolution of unity associated with the energy-momentum operator $P$ and if $\operatorname{supp}(\chi) \cap \bar{V}_{+}=\emptyset$ the right hand side of this equality vanishes. This fact shows that the spectrum of the energy-momentum operator $P$ is contained in $\bar{V}_{+}$.

Lastly we want to make mention of the cluster decomposition. If we add to the third axiom the statement that $\{p=0\}$ is an isolated eigenvalue of the energymomentum operator $P$ and the corresponding eigenspace is one-dimensional, then the cluster property holds: For a spacelike vector $a$ we have

$$
\int \mathfrak{B}_{n+m}\left(\underline{x}_{n}, \underline{y}_{m}+\lambda a\right) \phi\left(\underline{x}_{n}\right) \psi\left(\underline{y}_{m}\right) d \underline{x}_{n} d \underline{y}_{m} \rightarrow \mathfrak{W}_{n}(\phi) \mathfrak{W}_{m}(\psi)
$$

as $\lambda \rightarrow \infty$. The proof is carried out in the same way as in Jost [17]. The converse proposition that the cluster property implies the uniqueness of the vacuum is also proved in a way quite similar to tempered fields [1]. 


\section{References}

1. Streater, R.F., Wightman, A.S.: PCT, spin and statistics, and all that. New York-Amsterdam: Benjamin 1964

2. Jaffe, A.: Phys. Rev. 158, 1454-1461 (1967)

3. Constantinescu, F.: J. math. Phys. 12, 293-298 (1971)

4. Lomsadze, Yu. M., Krivsky, I. Yu., Shuba, Yo. M.: Kiev preprints ITP-72-62E 1972, ITP-74-130E 1974, ITP-74-134E 1974

5. Osterwalder, K., Schrader, R.: Commun. math. Phys. 31, 83-112 (1973); 42, 281-305 (1975) Osterwalder, K.: Euclidean Green's functions and Wightman distributions. In: Constructive quantum field theory, Lecture Notes in Physics, No. 25, pp. 71-93. Berlin-Heidelberg-New York: Springer 1973

6. Kawai, T.: J. Fac. Sci. Univ. Tokyo Sec. IA 17, 467-517 (1970)

7. Ito, Y., Nagamachi, S.: J. Math. Tokushima Univ. (to appear)

8. Constantinescu, F., Thalheimer, W.: Commun. math. Phys. 38, 299-316 (1974)

9. Gel'fand,I.M., Shilov, G.E.: Generalized functions, Vol. 2. New York-London: Academic Press 1968

10. Mityagin, B. S.: Trudy Moskov Mat. Obšč. 9, 317-328 (1960)

11. Ion,P.D.F., Kawai, T.: Theory of vector-valued hyperfunctions. RIMS preprint (1973)

12. Komatsu, H.: J. Math. Soc. Japan 19, 366-383 (1967)

13. Treves, F.: Topological vector spaces, distributions, and kernels. New York-London: Academic Press 1967

14. Sato, M., Kawai,T., Kashiwara, M.: Microfunctions and pseudo-differential equations. In: Proceedings of Katata conference 1971, Lectures Notes in Mathematics, No. 287, pp. 264-529. Berlin-Heidelberg-New York: Springer 1973

15. Morimoto, M.: J. Fac. Sci. Univ. Tokyo Sec. IA 17, 215-239 (1970)

16. Sato, M.: Sûgaku no Ayumi 15, 9-72 (1970) (in Japanese, Notes by Kashiwara)

17. Jost, R.: The general theory of quantized fields. Providence: Amer. Math. Soc. 1965

Communicated by A. S. Wightman

Received August 12, 1975; in revised form October 8, 1975 